\title{
In-situ IR Spectroscopy to Study Anodic Oxidation of Si(111) in KOH Solution
}

\author{
Harold G.G. Philipsen ${ }^{\mathrm{a}}$, Jean-Noël Chazalviel ${ }^{\mathrm{b}}$, Philippe Allongue ${ }^{\mathrm{b}}$, \\ François Ozanam ${ }^{\mathrm{b}}$, John J. Kelly ${ }^{\mathrm{a}}$ \\ ${ }^{a}$ Debye Institute, Condensed Matter and Interfaces, University of Utrecht, \\ P.O. Box 80000, 3508 TA, Utrecht, The Netherlands \\ ${ }^{\mathrm{b}}$ Physique de la Matière Condensée, Ecole Polytechnique, \\ CNRS, 91128 Palaiseau, France
}

Fourier Transform Infrared (FTIR) spectroscopy was used to study in-situ the anodic oxidation of n-type $\mathrm{Si}(111)$ in $\mathrm{KOH}$ solution. Changes in surface chemistry were followed during oxide growth. The results are considered on the basis of a model developed from electrochemical measurements.

\section{Introduction}

Chemical etching of silicon in alkaline solutions is a strongly anisotropic process (1). The variation in etch rate for the different crystallographic faces can be exploited to make a range of geometric forms required for micro-electromechanical systems (MEMS) (2). Silicon can also be oxidized electrochemically: when the potential of the semiconductor is made positive with respect to the open-circuit value, anodic current is observed for both p-type and n-type electrodes. In both cases the semiconductor eventually passivates; this process is widely used as an etch-stop mechanism in membrane fabrication for MEMS (2). As in etching, anisotropy is also observed in anodic passivation. Electrochemical experiments with a V-groove electrode geometry have shown that the kinetics of anodic oxidation of $\mathrm{Si}(111)$ are considerably slower than those of $\mathrm{Si}(100)$. The n-type (111) electrode is exceptional in that a considerable induction time is required for oxide formation in potentiodynamic and potential-step measurements (3).

In general, anodic oxidation of a p-type semiconductor involves valence-band holes. Anodic current from an n-type semiconductor, however, must result from electron injection into the conduction band $(3,4,5)$. Because of the surprisingly large current densities observed with n-type silicon in alkaline solution (the values are comparable to those for the corresponding p-type electrode), it was concluded that the electron-injecting states arise from the chemical etching reaction $(4,5)$. Anisotropy in electrochemical oxidation is therefore related to the stability of the surface with respect to chemical dissolution $(3,4,6)$. (The $(111)$ surface with monohydride termination and three $\mathrm{Si}-\mathrm{Si}$ back bonds is markedly more stable than the (100) surface with its dihydride termination and two back bonds.) The importance of 'chemical activation' is supported by the observation that the addition of a low concentration of an oxidizing agent $\left(\mathrm{H}_{2} \mathrm{O}_{2}\right)$ to the $\mathrm{KOH}$ solution greatly enhances the rate of anodic oxidation and subsequent passivation of n-type $\mathrm{Si}(111)$ (7). 
On the basis of macroscopic electrochemical measurements a qualitative model has been proposed for the passivation of n-type silicon $(3,4,5)$. Chemical etching consists of two main steps. Nucleophilic attack by $\mathrm{OH}^{-}$ions leads to hydrolysis of $\mathrm{Si}-\mathrm{H}$ surface bonds: surface $\mathrm{Si}-\mathrm{OH}$ and hydrogen gas are formed. The Si-OH polarizes the Si-Si back bonds, which are split by water. Intermediates of these two chemical reactions may inject electrons into the conduction band of the semiconductor $(3,4,5)$. This anodic process leads to a change in the reaction route. Instead of a regeneration of the hydride surface, oxide is formed which leads to passivation $(3,4,5,6)$. Electrochemical evidence is, however, indirect. On the other hand, spectroscopic techniques can provide direct information on the surface chemistry and, in certain cases, on charge distribution and surface states (8). In the present study we have combined potential-step measurements with in-situ FTIR spectroscopy to study the anodic oxidation of macroscopic n-type $\mathrm{Si}(111)$ electrodes in $6.0 \mathrm{M} \mathrm{KOH}$ solution at $40.0^{\circ} \mathrm{C}$. The relatively slow kinetics of this process allows us to follow in real time changes in surface chemistry.

In-situ IR spectroscopy has been used previously to study oxide formation on silicon in acidic fluoride solution (9). The technique has also been applied to hydrogen-terminated silicon in alkaline solutions. Haiss et al. were able to correlate the increase in anodic current of n-type and p-type $\mathrm{Si}(100)$ with a decrease in the integrated Si-H intensity (10). Current transients measured on stepping from open-circuit potential (OCP) to the passivation range showed oxide growth to be fast for both types of electrode. Rappich et al. concluded on the basis of IR measurements that n-type Si(111) etching in $0.5 \mathrm{M} \mathrm{KOH}$ solution at room temperature remains hydrogen-terminated, confirming that hydrolysis of $\mathrm{Si}-\mathrm{H}$ and $\mathrm{Si}-\mathrm{Si}$ bonds controls the kinetics of dissolution (11). On anodic polarization of the electrode, the Si-H absorption signal disappears at quite modest current densities. This previous work, restricted to the analysis of the $\mathrm{Si}-\mathrm{H}$ absorption, has not considered silicon passivation, the subject of the present paper.

\section{Experimental}

Samples were cut from n-type $\mathrm{Si}(111)$ wafers which were float-zone purified (phosphorus doped; resistivity 130-150 $\Omega . \mathrm{cm}$; thickness $0.5 \mathrm{~mm}$; double side polished). Internal reflection prisms with polished $45^{\circ}$ bevelled edges were used. Typical dimensions of the samples were $20 \times 15 \mathrm{~mm}$. Before use, the samples were cleaned in a 'piranha' solution (3:1 mixture of concentrated $\mathrm{H}_{2} \mathrm{SO}_{4}$ and $30 \% \mathrm{H}_{2} \mathrm{O}_{2}$, immersion time 10 minutes) and then thoroughly rinsed with doubly distilled water. Beakers and tweezers made of PFA (Savillex, USA) were used for cleaning and handling.

The native oxide layer was removed in an oxygen-free $40 \% \mathrm{NH}_{4} \mathrm{~F}$ solution. This etchant is known to yield ultraflat $\mathrm{Si}(111)$ surfaces if the solution is oxygen-free and the sample is 'cathodically protected' by the creation of a sacrificial anodic area $(12,13)$. Before preetching, the (111) surface is terraced with a step density determined by the substrate miscut. Subsequently, the surface was exposed to the $\mathrm{KOH}$ solution and IR spectroscopic measurements were performed.

The upper part of figure 1 shows a schematic representation of the electrochemical cell used for the experiments (more details can be found in the figure caption). The sample was pressed on the cell with an O-ring (Viton, FPM, $10.00 \times 1.00 \mathrm{~mm}$ ). Due to this geometry, ten useful reflections were obtained at the 'wetted' interface. The etchant was 
prepared from 'VLSI grade' KOH (Merck, Selectipur, \# 1.05019, used as received) and doubly distilled water. The solution was deaerated using nitrogen.

Infrared spectra were acquired with a Bruker Equinox-55 spectrometer over a spectral range of $950-4000 \mathrm{~cm}^{-1}$ (limited by bulk Si absorption) with a resolution of $4 \mathrm{~cm}^{-1}$. Reference spectra were taken under cathodic polarization during each measurement cycle. To increase the signal-to-noise ratio, spectra were recorded during multiple cycles and co-added. By polarizing the IR beam, additional information could be obtained about the orientation of the absorbing dipoles. An s-polarized beam is sensitive to absorption modes parallel to the surface. In the case of p-polarized radiation both parallel and perpendicular components are detected. Therefore, a perfectly flat Si(111) surface with a very small miscut is expected to show only absorption in p-polarization for the silicon monohydride, as this bond is perpendicular to the terrace surface. This gives a qualitative indication of the surface flatness under etching conditions.

In previous work with V-groove electrodes, the anodic passivation of n-type $\mathrm{Si}(111)$ facets was studied by measuring current transients after the potential was stepped from open-circuit to a value in the passive range (3). Allongue and co-workers have shown with scanning tunneling microscopy (STM) that the $\mathrm{Si}(111)$ surface in hydroxide solution is susceptible to 'pitting' at open-circuit potential (6). In order to prevent surface roughening in the active etching range, a potential negative with respect to OCP was maintained. After passivation of the surface with anodic oxide, the potential was switched back to a cathodic value and oxide etch back was followed by measuring current as a function of time.

\section{Results and Discussion}

Figure 2(A) shows typical current transients measured during anodic and cathodic cycles. At positive potential, the hydrogen-terminated surface is anodically oxidized, as witnessed by the small anodic current peak. When the potential is switched to a negative value, a cathodic current due to hydrogen evolution is observed. This current gradually increases as the anodic oxide is etched back. Finally, a steady-state current is established.

Figure 2(B) shows the current transient for the anodic cycle in more detail. When the potential is stepped to a value in the passive range an anodic current is observed that rapidly drops to a low level. This process is very likely due to passivation of step edges. Then the current slowly increases and passes through a maximum, after which the surface is passivated. Such anodic transients were reproducible and were similar to transients measured with n-type (111) facets (3). Previous experiments have shown that the time required to passivate the surface, i.e. to reach current maximum, decreases strongly with increase in temperature. 

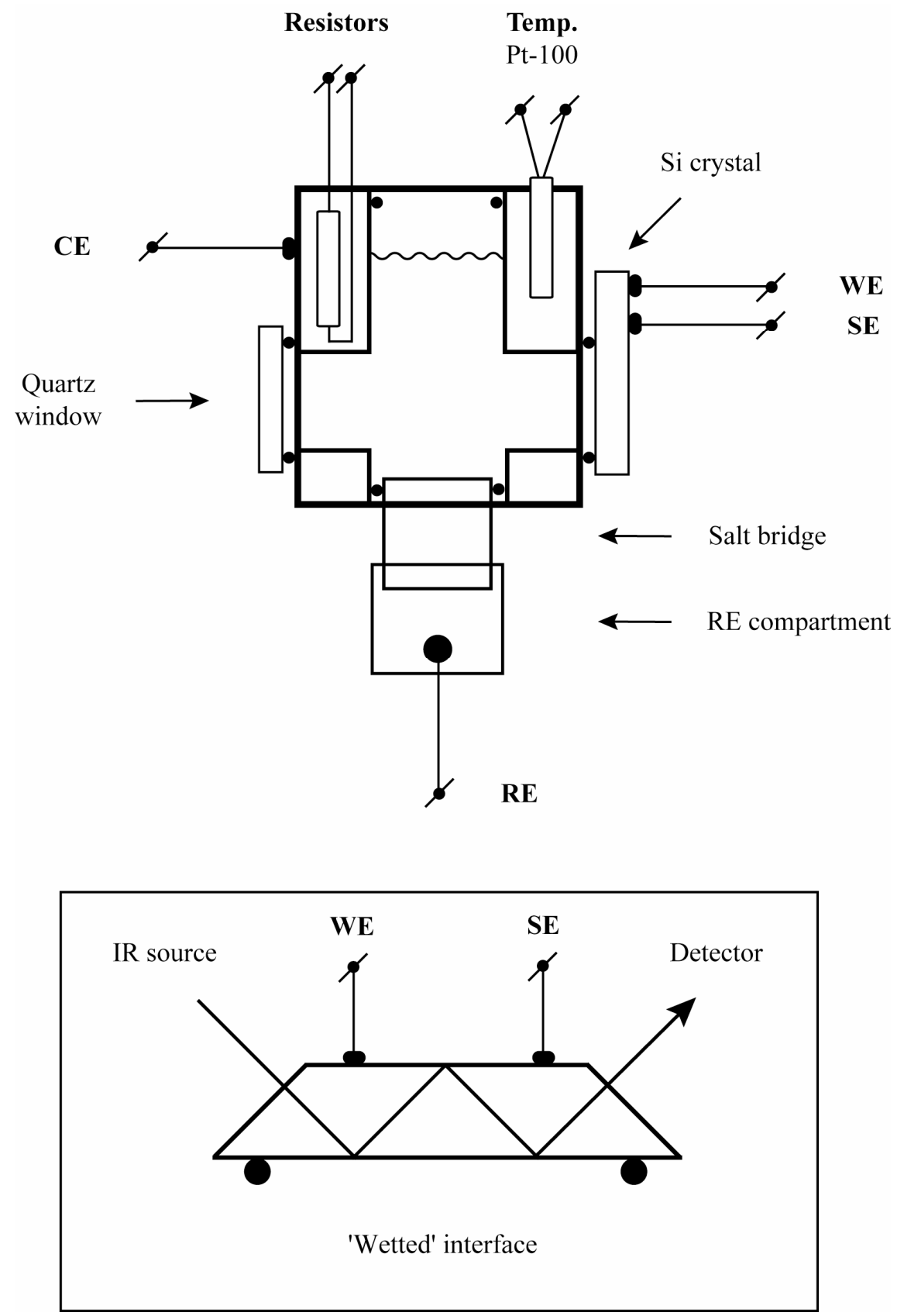

Figure 1. (Upper part) Schematic representation of the in-situ cell in side view. The volume of the liquid in the cell is $\sim 2.5 \mathrm{~mL}$. Thermostating was achieved by resistive heating and a temperature sensor with a control unit. The reference electrode (RE, $\mathrm{Hg} / \mathrm{HgO}$ ) was attached to the cell via a salt bridge and the stainless steel body of the cell formed the counter electrode (CE). Two contacts were made to the Si crystal. The electrical current flows through the working electrode (WE) connection and the 'sensing' electrode (SE) was employed for measuring the potential. A four-probe potentiostat was used to avoid problems due to the high resistivity of the silicon. All measurements were performed in the dark. (Lower part) Top view of a Si crystal with multiple internal reflections. The WE and SE contacts were made in the two upper corners of the sample, well outside the plane in which the internal reflections occur. 

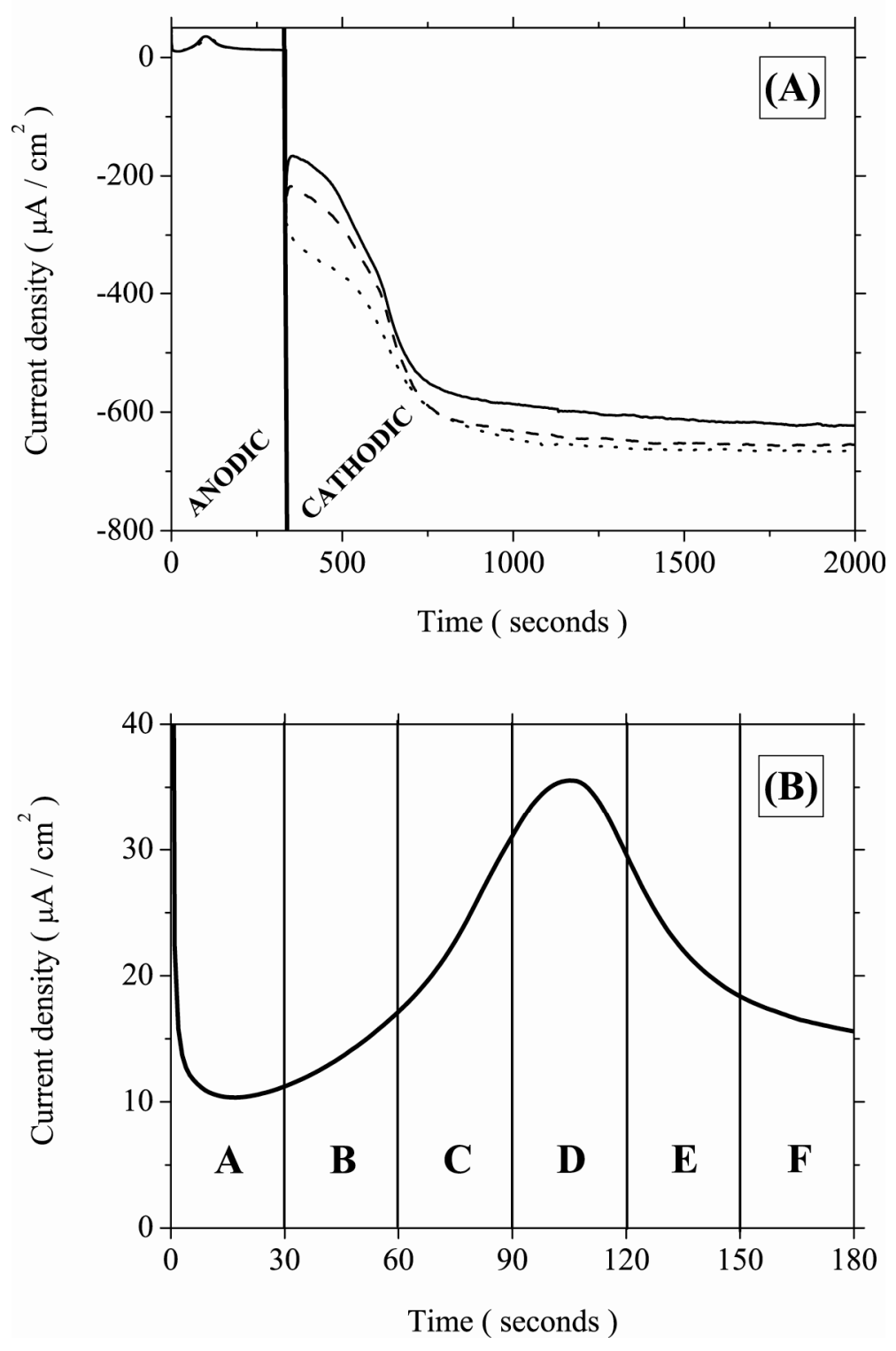

Figure 2. (A) Current transients for three cycles. During anodic polarization, oxide is nucleated and grows. The current maximum indicates the onset of passivation. During the cathodic cycle, the oxide dissolves. (B) Enlargement of one of the anodization transients. The capital letters at the bottom of the figure indicate the time interval during which the corresponding IR spectra shown in Fig. 3 were measured. Conditions: $\mathrm{n}-\mathrm{Si}(111)$ in contact with $6.0 \mathrm{M} \mathrm{KOH}$ at $40.0{ }^{\circ} \mathrm{C}$.

Figure 3(A) shows a series of spectra measured during an anodic oxidation cycle. In these spectra, features from silicon monohydride $\left(2062 \mathrm{~cm}^{-1}\right)$, silicon oxide $\left(1000-1250 \mathrm{~cm}^{-1}\right.$ 
region), and electrolyte-related absorptions $\left(1640 \mathrm{~cm}^{-1}\right.$ and region of $\left.3000-3500 \mathrm{~cm}^{-1}\right)$ are present.

During anodic oxidation, the $\mathrm{Si}-\mathrm{H}$ absorption shows a continuous decrease with time. When the current transient reaches its maximum, i.e. during the acquisition of spectrum $\mathrm{D}$, the $\mathrm{Si}-\mathrm{H}$ absorption has almost completely disappeared (for the fully oxidized state, the change in peak absorbance amounts to $-7 \times 10^{-4}$ ). Due to interaction between the electrolyte and the $\mathrm{Si}-\mathrm{H}$ species, this absorption is broadened (full width at half maximum is approximately $\left.40 \mathrm{~cm}^{-1}\right)(14)$.

The growth of oxide on the electrode is indicated by the appearance of two distinct features in the wavenumber range $1000-1250 \mathrm{~cm}^{-1}$ (see figure 3(B) for an enlargement of this region). They are unambiguously ascribed to the TO mode (at low energy) and LO mode (at high energy) of the SiOSi asymmetric stretch vibration. In the initial stage of oxidation only the TO mode, which is centered at $1030 \mathrm{~cm}^{-1}$, is present. On further oxide growth, the intensity of the TO feature continues to increase and reaches a maximum value just after the current has peaked (see spectrum E). Later in oxide growth, the LO peak appears at higher wavenumbers, shifts towards higher wavenumbers when the electrode becomes passivated and finally stabilizes at $1185 \mathrm{~cm}^{-1}$. At low oxide coverage, the LO-mode is not significant and it slowly increases during anodization. This is related to the way the oxide grows; the oxide is initially nucleated at step edges and the surface coverage is therefore small. Subsequently, oxide nucleation on the terraces becomes significant. With the presence of these oxide islands, neighbouring $\mathrm{Si}-\mathrm{Si}$ bonds become destabilized. This effect accelerates the oxidation process and the oxide 'spreads' over the surface.

As the oxide grows, the optical reflection takes place further off the electrolyte, resulting in a decrease in electrolyte absorbance. As this absorption is much stronger than the absorption of surface species, electrolyte-related features show up in the spectra (8). The absorption of the water bending (scissor) mode at $1635 \mathrm{~cm}^{-1}$ gradually decreases with time. In the $3000-4000 \mathrm{~cm}^{-1}$ range the absorbance of various $\mathrm{OH}$ species results in a more complex shape. Two contributions, $\mathrm{OH}$ groups of water and surface $\mathrm{OH}(\mathrm{Si}-\mathrm{OH})$, are superimposed. During oxidation, the electrolyte-related absorption continuously decreases, resulting in a negative going feature that arises at approximately $3300 \mathrm{~cm}^{-1}$. Concomitant with the growth of oxide is the appearance of the Si-OH absorption. The growth of oxide is 'nucleated' by the formation of $\mathrm{OH}$ groups at the surface, leading to the oxidation of Si-Si backbonds. Si-OH species remain present even when the electrode is fully passivated.

A noticeable aspect in this series of spectra is the change in background, which levels off as the surface becomes passivated with an oxide layer. There is a strong correlation between the change in background and the progress of anodic oxidation: the change in background occurs mainly in the time span up to the current peak. This very broad, tailing background is related to the IR absorption by surface states (15). The negative sign indicates a decrease in surface-state absorption. This decrease may be due either to a change in population or to a disappearance of the states, the long time constant favoring the latter interpretation. Likely candidates for such electronic states are those that arise during chemical etching of the surface or (more plausibly) those related to hydrogen evolution and incorporation into the silicon lattice. 
We have shown results only for p-polarization and discussed the changes in the spectra for anodic oxidation. During the oxide etch back under cathodic polarization, it was observed that the $\mathrm{Si}-\mathrm{H}$ absorption re-appears and that the oxide related features gradually disappear due to the chemical dissolution.

Surface roughening is an important factor that has to be considered, as this can affect the information extracted from this type of experiments. By measuring anodization spectra with s-polarized light we confirmed that the Si-H absorption and the LO mode for the oxide are not present. This proves that the surface remains flat under these conditions.
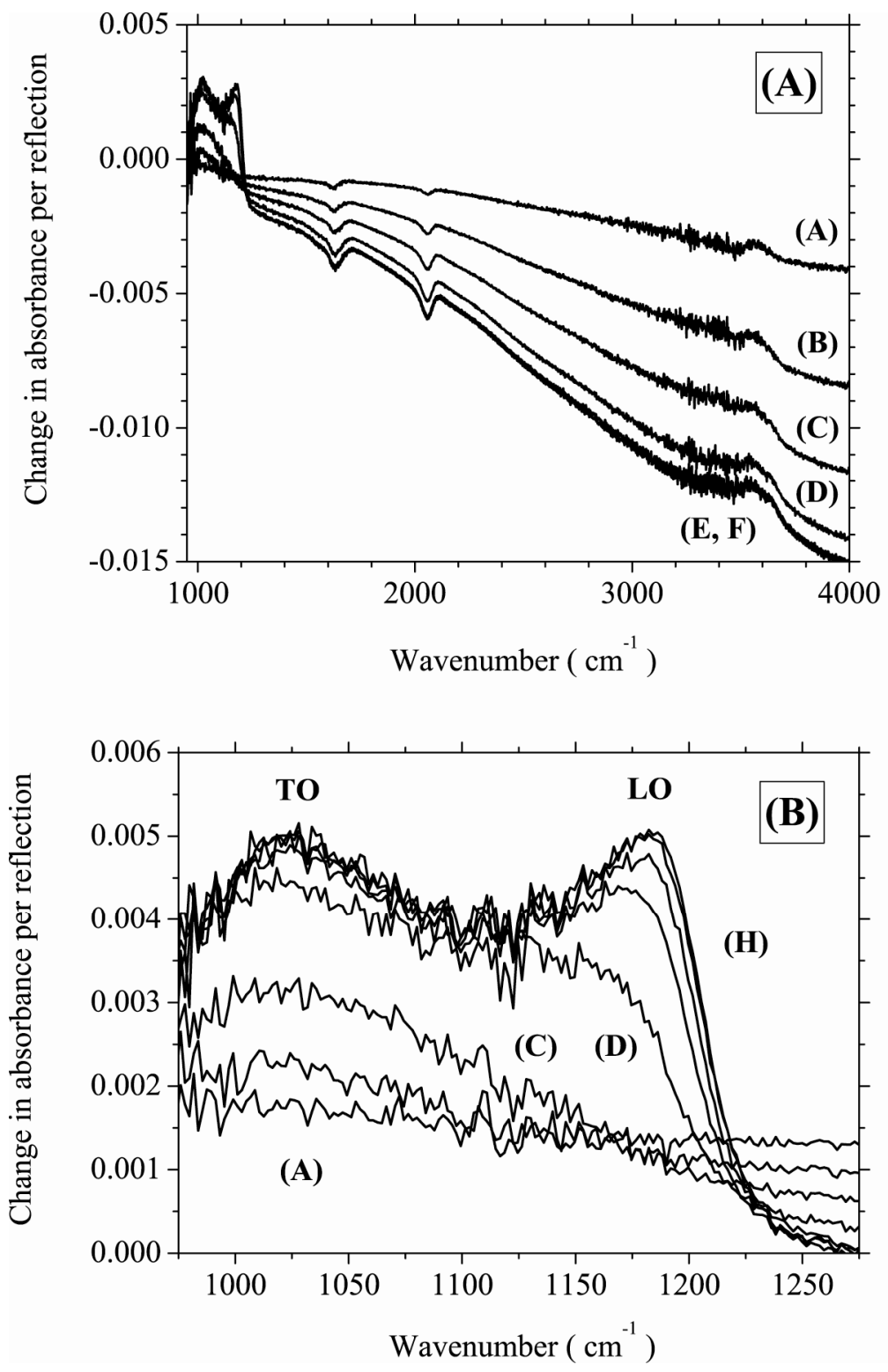

Figure 3. (A) Spectra measured in p-polarization during an anodic oxidation cycle. Time increases on going from curve $(\mathrm{A})$ to $(\mathrm{F})$ (see figure 2(B)). The spectra are referenced to spectra measured under cathodic polarization. A negative peak indicates disappearance of a species, a positive peak its appearance. (B) Detail of the silicon oxide region. 


\section{Conclusions}

Using in-situ FTIR spectroscopy, we have studied the changes in surface chemistry during the anodic oxidation of $\mathrm{Si}(111)$. Due to the chemical stability of the surface, this process is very slow and the nucleation and subsequent growth of the oxide on the surface could thus be studied. During anodic oxidation, the $\mathrm{Si}-\mathrm{H}$ termination is gradually replaced by $\mathrm{Si}-\mathrm{OH}$ and the oxide is nucleated. In the initial stage of anodic oxidation, step

edges are passivated. Subsequently, oxide nucleation and growth on the terraces can also occur.

\section{Acknowledgments}

Fouad Maroun (Labo-PMC, Ecole Polytechnique, France) is thanked for his help with the experiments. This work was carried out with a Marie Curie Fellowship from the European Union (Contract HPMT-CT-2001-00328) and financially supported by the Dutch Technology Foundation (STW, TPC-5990).

\section{References}

1. R.A. Wind, H. Jones, M.J. Little and M.A. Hines, J. Phys. Chem. B, 106, 1557 (2002).

2. M. Elwenspoek and H. Jansen, In: Silicon Micromachining, Cambridge studies in semiconductor physics and microelectronic engineering - Volume 7; Cambridge university press: Cambridge, 1998.

3. H.G.G. Philipsen and J.J. Kelly, J. Phys. Chem. B, 109, 17245 (2005).

4. P. Allongue, V. Costa-Kieling and H. Gerischer, J. Electrochem. Soc., 140, 1018 (1993).

5. X. Xia, C.M.A. Ashruf, P.J. French, J. Rappich, J.J. Kelly, J. Phys. Chem. B, 105, $5722(2001)$.

6. P. Allongue, V. Costa-Kieling and H. Gerischer, J. Electrochem. Soc., 140, 1009 (1993).

7. H.G.G. Philipsen and J.J. Kelly, To be published.

8. J.-N. Chazalviel, B.H. Erné, F. Maroun and F. Ozanam, J. Electroanal. Chem., 509, 108 (2001).

9. R. Outemzabet, M. Cherkaoui, N. Gabouze, F. Ozanam, N. Kesri and J.-N. Chazalviel, J. Electrochem. Soc., 153, C108 (2006).

10. W. Haiss, P. Raisch, D.J. Schiffrin, L. Bitsch and R.J. Nichols, Faraday Discuss., 121, 167 (2002).

11. J. Rappich, H.J. Lewerenz and H. Gerischer, J. Electrochem. Soc., 140, L187 (1993).

12. P. Allongue, C. Henry de Villeneuve, S. Morin, R. Boukherroub and D.D.M. Wayner, Electrochimica Acta, 45, 4591 (2000).

13. S.P. Garcia, H. Bao, M. Manimaran and M.A. Hines, J. Phys. Chem. B, 106, 8258 (2002).

14. A. Venkateswara Rao, F. Ozanam and J.-N. Chazalviel, J. Electrochem. Soc., 138, 153 (1991).

15. J.-N. Chazalviel, A. Venkateswara Rao, J. Electrochem. Soc., 134, 1138 (1987). 